\title{
Brasil y sus intereses \\ en la construcción de Cachuela Esperanza, Bolivia
}

\author{
Costa, A.K.N., \\ Universidade Federal do ABC, Santo André, Brasil. \\ Email: keiko.nishida@ufabc.edu.br \\ Vibian, C.F., \\ Universidade Federal do ABC, Santo André, Brasil. \\ Email: carolina.vibian@ufabc.edu.br \\ Cardoso, D.E.V. \\ Universidade Federal do ABC, Santo André, Brasil. \\ Email: dcardoso@aluno.ufabc.edu.br

\section{Guerra, S.M.G.} \\ Universidade Federal do ABC, Santo André, Brasil. \\ Email: sinclair.guerra@ufabc.edu.br
}

Resumen: La necesidad de energía obliga a los países a reflexionar sobre las formas de obtener este recurso más limpio y más barato. Como alternativa a esto se promueven intentos de integración entre los diferentes países. El Complejo Madeira tiene un gran potencial para la producción de energía hidroeléctrica, como resultado de caídas y rápido flujo de agua en los afluentes del río Madeira, en especial el río Beni, donde se planea construir una central hidroeléctrica en Cachuela Esperanza, como resultado de la alianza entre Brasil y Bolivia. El proyecto hidroeléctrico está rodeado por controversias y afecta la imagen de Brasil pues éste puede ser considerado como un país oportunista, ya que la mayoría de los impactos negativos de la construcción no se exportarán, y el país se beneficiará de la venta de energía a bajo costo por Bolivia.

Palabras clave: Cachuela Esperanza, hidroeléctricas, energía, sedimentos.

\section{Brazil and its interests in the construction of Cachuela Esperanza, Bolivia}

\begin{abstract}
The energy requirement obliges countries to think on ways to get this resource cleanly and cheaply. Alternative to this are the attempts of integration between different countries. The Madeira Complex has great potential for hydroelectric generation as a result of falls and rapid flow of water in the tributaries of the Madeira river, especially the Beni river, where building a hydroelectric plant in Cachuela Esperanza is planned, as a result of the partnership between Brazil and Bolivia. The hydroelectric project is surrounded by controversy and affects the
\end{abstract}


image of Brazil as opportunistic, since most of the negative impacts resulting from the construction will not be exported, and the country will benefit from the sale of low-cost energy through Bolivia.

Key words: Cachuela Esperanza, hidropower, energy, sediments.

\section{Brasil e seus interesses na construção de Cachuela Esperanza, Bolívia}

Resumo: A necessidade de energia exige aos países a refletir sobre as formas de obter esse recurso mais limpo e mais barato. Como alternativa a isto se promovem esforços de integração entre os diferentes países. O Complexo Madeira tem um grande potencial para a produção de energia hidroelétrica, como resultado de quedas e rápido fluxo de água nos afluentes do rio Madeira, em especial o Rio Beni, onde se planeja construir uma usina hidrelétrica em Cachuela Esperanza, como resultado da aliança entre Brasil e Bolívia. O projeto da hidrelétrica é cercada de polêmicas e afeta a imagem do Brasil, pois este país pode ser considerado como um país oportunista, já que a maioria dos impactos negativos da construção não será exportada, e o país se beneficiará com a venda de energia a baixo custo por parte de Bolívia.

Palavras-chave: Cachuela Esperanza, hidrelétricas, energia, sedimentos.

\section{Introducción}

La energía es considerada como un factor importante en el desarrollo de nuestro planeta, porque está estrechamente relacionada con la historia de la humanidad y está presente en prácticamente todos los sectores de la sociedad: el trabajo, la economía, la política y el medio ambiente influyendo directamente en nuestros comportamientos diarios (HINRICHS, KLEINBACH Y REIS, 2010)

La energía utilizada por el hombre recibe el nombre de energía secundaria, puesto que se origina a partir de la transformación de la energía primaria. Por lo tanto las fuentes de energía primaria y secundaria pueden ser clasificadas como renovables y no renovables. Las fuentes renovables son esas respuestas en un corto período de tiempo, es decir, antes de su consumo total, mientras que los recursos no renovables son aquellos en los que la sustitución de sus recursos no ocurre en un periodo corto de tiempo. Los combustibles fósiles son claros ejemplos, porque se consumen antes de que se puedan reponer en la naturaleza (GOLDEMBERG y LUCON, 2008).

Ejemplos de fuente de energía renovable muy representativo en América Latina son las hidroeléctricas que se destacan gracias al potencial hídrico de la región. De acuerdo con el criterio de la disponibilidad hídrica por habitante de la ONU en 1997, se consideran países "ricos" a los que están en el rango de 10.000 a 100.000 m3/persona/año. Se encuentran en 
esta situación: Argentina, Bolivia, Brasil, Chile y Venezuela (PORTOGONÇALVES, 2008).

Sin embargo, las hidroeléctricas generan una serie de impactos aun siendo consideradas renovables. En este contexto, el presente documento tiene la finalidad de presentar el Complejo Madeira enfocado en el proyecto de la hidroeléctrica de Cachuela Esperanza - planta hidroeléctrica que será construida en Bolivia y Brasil, manifestando ciertos intereses en este proyecto.

\section{Las centrales hidroeléctricas y sus impactos}

Antes de la construcción de la usina hidroeléctrica es realizado un estudio de planificación del sector energético, entre lo que se investiga, lugar de la construcción, posibles impactos ambientales generados por la ejecución del proyecto, y el potencial hidroeléctrico de la cuenca, con el fin de definir el mejor lugar de la caída de agua. (PIRES, 2001; LIMA, 2009).

Después del estudio se analiza la viabilidad económica del proyecto y comienza el proceso de licitación para su ejecución. Una vez completados estos pasos se da lugar al inicio de la construcción de la obra.

Aunque es considerado por muchos como una fuente "limpia", es preciso un análisis crítico sobre esta fuente, tomándose en cuenta los impactos ambientales generados por la misma. De acuerdo con la Resolución 001 del 23 de enero de 1986, artículo 1 e inciso I al V de CONAMA (Consejería de Medio Ambiente de Brasil) significará impacto ambiental cualquier cambio que afecte directa o indirectamente las actividades de las personas y al medio ambiente (BRASIL, 1986).

Souza (2000), señala algunos elementos que justifican por qué este tipo de fuente ha demostrado ser insustentable en todo el mundo: los cambios físicos de los ríos, como cambios en la corriente, temperatura, poco movimiento del agua embalsada que causan condiciones anóxicas, con efectos negativos sobre las especies, y conduce a la pérdida de la biodiversidade. Además de estos factores, existe todavía el problema de los gases de efecto invernadero (GEI), considerados como responsables del calentamiento global y el cambio de calidad de las masas de agua - potenciales generadores de los problemas de salud pública, con la proliferación de vectores transmisores de enfermedades (BERMANN, 2007).

Anexo a lo anterior hay otros problemas que se refieren a la necesidad de locomoción de las personas, es necesario que una gran área sea utilizada para la construcción del embalse (lago artificial). Los habitantes de la costa, además de perder sus bienes materiales sufren hasta con la pérdida de espacios simbólicos, tales como iglesias y lugares sagrados para las comunidades (PINHEIRO, 2007). 


\section{Complexo Madeira}

Durante una reunión entre presidentes de América del Sur en agosto de 2000, llega la IIRSA (Iniciativa para la Integración de la Infraestructura Regional en América del Sur), en la cual fue discutida la idea de coordinar el planeamiento para la construcción de infraestructura de los países sudamericanos. De esta forma, la Iniciativa para la Integración de la Infraestructura Regional Suramericana (IIRSA) coordina los gobiernos, instituciones financieras multilaterales y el sector privado, para que pueda controlar los planes y programas de inversión, priorizar los ejes de integración y el desarrollo en los sectores de transporte, energía y telecomunicaciones (SUAREZ, GUERRA, UDAETA, 2006).

El complejo hidroeléctrico del Madeira corresponde al mayor grupo de proyectos de la IIRSA y está situado en el eje Perú-Brasil-Bolivia, correspondiendo más específicamente al sur de Perú, en la región amazónica de Bolivia y el noroeste de Brasil (FOBOMADE, 2008).

Existen en el eje Perú-Brasil-Bolivia tres grupos de proyectos, cuyo objetivo es unir físicamente las tres regiones a los puertos del Pacífico y también a los grandes centros de consumo por medio terrestre y fluvial, de forma que pueda ser conectado este eje con otros.

Los tres grupos corresponden a la ejecución de proyectos de corredores diferentes:

- "El Corredor Porto Velho-Río Branco-Pto Assis-Pto MaldonadoCusco/Juliaca-Puertos marítimos del Pacífico.

- El Corredor Norte La Paz-Guayaramerin-Cobija Río Branco, que vincula los Estados de Rondonia y Acre (Brasil) a través de las rutas Guayaramerín - Riberalta - La Paz y Río Branco - Cobija - Riberalta con el Eje Interoceánico Central y los puertos del Pacífico.

- El Corredor de Integración Fluvial Madera, Madre de Dios, Beni, que incluye la construcción de centrales hidroeléctricas y de esclusas para la navegación del río Madera” (FOBOMADE 2008:1). 


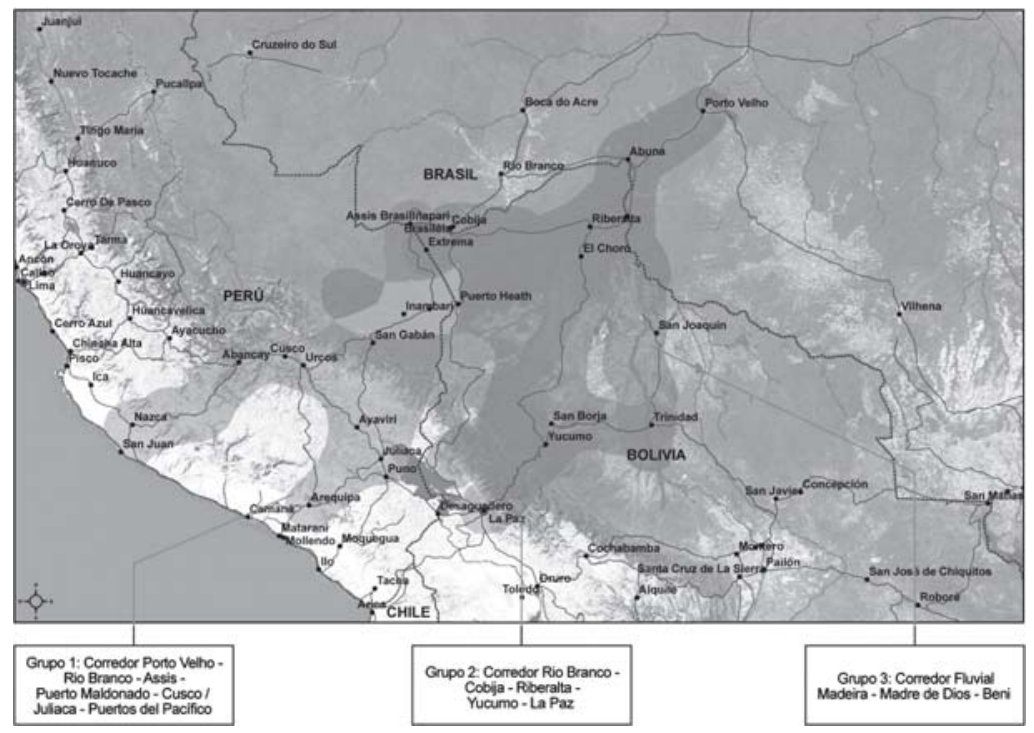

\section{Figura 1. Grupos de proyectos del eje Perú-Brasil-Bolivia.}

Fuente: FOBAMADE, 2008

También forma parte del complejo Madeira las represas de Santo Antônio, de Jirau y las líneas de transmisión entre las dos represas bien como navegación entre las ciudades de Porto Velho, Guajará-Mirim en Brasil y Guayaramerín en Bolivia. Además de los proyectos de navegación por el río Beni y por el río Madre de Dios en Perú (COLLAZOS, AZTARAIN, AUBAREDA, 2010).

Estos proyectos son posibles gracias al río Madeira, que tiene sus orígenes en la Cordillera de los Andes, y que está formado por los ríos Beni, Madre de Dios, que se añaden posteriormente a los ríos Marmoré e Itnez, que al alcanzar el Amazonas se convierte en uno de los cinco ríos más caudalosos del mundo, llevando, en su flujo, una gran carga de sedimentos. También es importante saber que el río Madeira, en la parte boliviana tiene muchos afluentes y que estos tienen potencial para la generación de energía hidroeléctrica, siendo que su abundancia y régimen hidrológico dependen, sobre todo de los ríos Beni y Marmoré (FOBOMADE, 2008).

\section{Cachuela Esperanza}

En La década de los años 80 la Empresa Nacional de Electricidad de Bolivia (ENDE), realizo sus primeros estudios sobre la posibilidad de cons- 
truir una represa en Cachuela Esperanza. A partir de esta fecha, el proyecto de construir la hidroeléctrica de Cachuela Esperanza resurgió como una prioridad en los diferentes gobiernos de Bolivia y a partir del año 2000 el proyecto comenzó a formar parte de la Iniciativa para la Integración de la Infraestructura Regional en América del Sur (IIRSA) y también del Complejo Madeira en conjunto con las represas de Santo Antônio y Jirau (CEADESC, 2011, MORAES, 2011).

En el 2008, el presidente de Bolivia, Evo Morales, contrató una empresa canadiense TECSULT-AECOM para realizar estudios y finalmente concluir el proyecto de la hidroeléctrica (ARISMENDI, 2010). Los estudios fueron completados en noviembre del 2009 y la TECSULT -AECOM anunció que el proyecto Cachuela Esperanza era económicamente viable, desde que parte de la energía fuera exportada para Brasil.

La hidroeléctrica tendría la capacidad para generar 990 MW con una superficie alargada de $690 \mathrm{Km}$. Las obras de construcción durarían de 7 a 8 años y requerirían de una inversión de dos millones de dólares.

Los impactos ambientales que se prevén son: variaciones en la calidad del agua, devastación de la vegetación, alteración en la migración de peces y aves (TECSULT AECOM, 2009), además de otros impactos que surgirán después de la construcción de las usinas, entre los que se destaca la emisión de gases del efecto invernadero, sabiendo que algunos estudios reportan que las plantas hidroeléctricas en el Amazonía producen más gases de efecto invernadero que las centrales termoeléctricas, debido a la descomposición de la vegetación del área que fue invadida por el agua y a las altas temperaturas, que proporcionan la emisión de metano (CEADESC, 2011).

Los impactos sociales también son previstos y corresponden a los desplazamientos y reubicación de la población, pérdida de tierras agrícolas, áreas de valor cultural, bienes y recursos naturales para la producción económica y el aumento de las enfermedades relacionadas con las obras (TECSULT AECOM, 2009).

Según el IIRSA, un gran número de comunidades indígenas y campesinas se verán afectadas, aproximadamente tres cuartas partes de la población rural. No hay un número oficial, pero se estima que 15 aldeas costeras de Bolivia puedan resultar damnificadas (LAATS, 2010).

El Banco Nacional de Desarrollo Económico y Social de Brasil (BNDES) será responsable por financiar la obra, se estima que el costo de la energía generada en Cachuela Esperanza será de \$ $65 \mathrm{MW} / \mathrm{h}$, frente a los \$ $43 \mathrm{MW} / \mathrm{h}$ de energía generada en Jirau. De este modo se obtendrá difícilmente un precio justo por la energía vendida a Brasil (SOTO, 2011).

El gobierno boliviano considera que la construcción de la hidroeléctrica será capaz de impulsar y fortalecer la economía de la región, convirtién- 
dose en una salida a la pobreza de la Amazonía Boliviana, proporcionando electricidad a las poblaciones ubicadas en el extremo norte del país, aunque existan muchos cuestionamientos sobre si esta será la mejor manera de promover el desarrollo de una región, con alto costo no solamente en el contexto económico sino también en el contexto social y ambiental (LAATS, 2009).

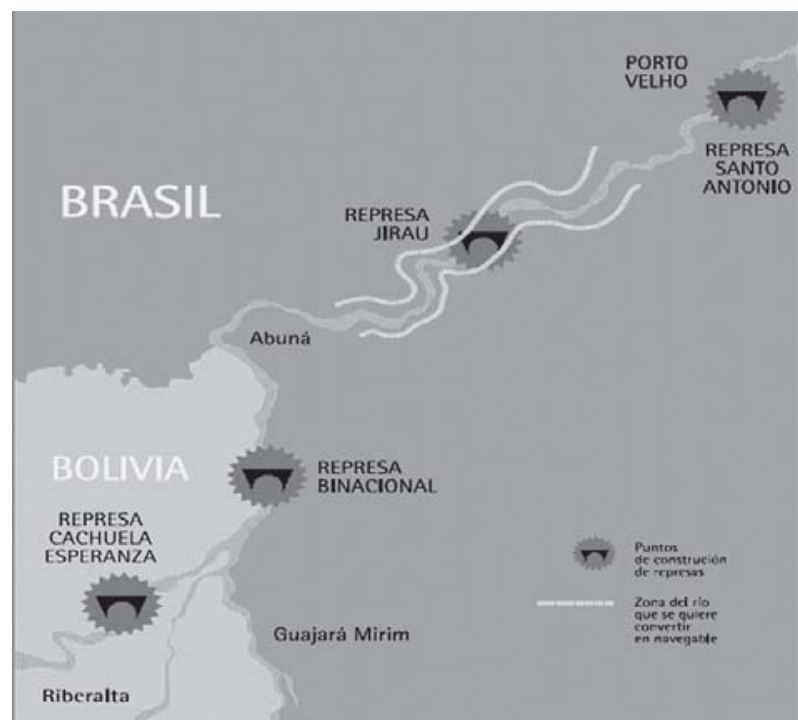

Figura 2. Lugar de represas en el Complejo Madeira.

Fuente: COLLAZOS, AZTARAIN, AUBAREDA, 2010.

Por otra parte, la política boliviana pretende garantizar el acceso a la energía de forma soberana, sustentable y socialmente justa, con las siguientes estrategias:

- Desarrollo de la estructura eléctrica para satisfacer la demanda interna y exportar el excedente generado;

- La soberanía y la independencia energética;

- Incrementar la cobertura de electricidad en las zonas urbanas y rurales;

- Consolidar la participación del Estado en el desarrollo de la industria eléctrica con soberanía y la igualdad social (MOURA, LEGEY y PEREIRA JÚNIOR, 2012). 


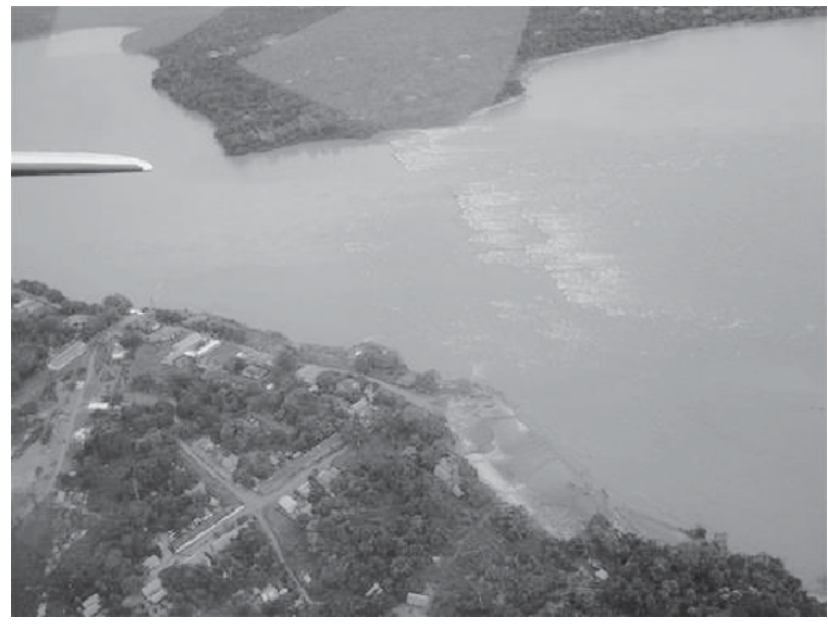

Figura 3. Cachuela Esperanza del área de visualización.

Fuente: Bank International Center de 2010.

\section{Cachuela Esperanza y sedimentos}

La hidroeléctrica Cachuela Esperanza tiene el potencial de suministrar a todo el territorio boliviano y todavía proveer energía a los países vecinos. Este proyecto tiene como objetivo no solamente desarrollar las regiones aisladas de la Amazonía boliviana, sino al conjunto reduciendo el costo de la energía que es alto en relación al Sistema Interconectado Nacional (SIN), que tiene algunos problemas como la poca cobertura (suministro de electricidad), poca infraestructura para el crecimiento y mala calidad de la oferta (ENDE, 2007). Otra ventaja en la construcción de la hidroeléctrica sería la posibilidad de convertir al río Madeira en un río navegable con esclusas o canales en las usinas.

La ubicación del proyecto se encuentra en el río Beni, que es uno de los ríos que ayuda a la formación del río Madeira y su naciente se encuentra en la Cordillera de los Andes. Esta región está formada por rocas areniscas que son quebradizas y tiene una alta tasa de precipitación con la geología local que con todas estas características contribuyen para la erosión, es decir, una alta producción de sedimentos (PCE, 2005; TUCCI, 2007). El flujo de sedimentos a la salida de los Andes que alcanza el río Beni es 191 X 106 t año-1 (GUYOT et al.1988), aproximadamente el 72\% del sedimento que llega hasta el río Madeira es proporcionado por el río Beni y el 28 \% proviene del río Marmoré (GUYOT, JOUANNEAU y WASSON, 1999).

Como el río Madeira es una de las principales fuentes de sedimentación de la cuenca Amazónica, es importante llevar a cabo un estudio de 
reposición de sedimentación en los embalses, con el fin de prolongar la vida de ellos (CARPIO, 2008), pues el proceso de obstrucción tiene varios impactos para el depósito como pérdidas en la producción de energía debido a la disminución en el almacenamiento del embalse, daño a las turbinas, erosión de la zona y de los márgenes próximos a la represa, riesgo de surgimiento de acumulación de arena o formación islas (PCE,2005).La composición media del sedimento es de $25 \%$ de arcilla, $60,6 \%$ de limo, $12 \%$ de arena, $12 \%$ de arena fina y $2,4 \%$ arena media, gruesa y grava (COBRAPE, 2006).

La concentración de sedimentos en suspensión del río Madeira se mantiene elevada durante la mayor parte del año y aumenta en temporada de inundaciones. Los estudios que fueron realizados por la empresa Furnas Centrales Eléctricas, concluyeron que gran parte del sedimento se mantendría retenido en las hidroeléctricas de Jirau y Santo Antônio, sin la construcción de las hidroeléctricas quedarían detenidos naturalmente el $40 \%$ de los sedimentos transportados. Con las construcciones esa cifra sube a un 84\%, la capacidad de los depósitos en la retención de los sedimentos es de sólo el 10\% para Santo Antônio y 5\% para Jirau (LANZA y ARIAS, 2011). La carga de sedimentos es de aproximadamente $720 \mathrm{MG} / \mathrm{l}$ pero puede llegar hasta 3500 MG/l durante los desbordamientos y mínimamente a 120 MG/l bajo. La descarga sólida en la región de las hidroeléctricas de Santo Antônio y Jirau es de 1.621.024 t/día y de 1.594.529 t/día, respectivamente.

Los cálculos realizados para la hidroeléctrica de Jirau con un nivel de agua de 87 metros sobre el nivel del mar, en un período de 39 años con la sedimentación, el volumen del depósito se reduce en $29,5 \%$ en caso de que ese nivel de agua fuera de 90 metros en un plazo de 45 años, habría una disminución de 51,6\% del volumen del depósito (COBRAPE, 2006).

Para Santo Antônio con un nivel de agua de 70 metros, la pérdida de $50 \%$ del volumen del depósito puede ocurrir en 74 años, por eso un muro de contención sería construido para evitar que los sedimentos lleguen a la turbinas y al canal de aducción (COBRAPE, 2006), pero de acuerdo a Fearnside (2007), debido a la incertidumbre del muro de contención que parece relativamente pequeño, los sedimentos tienden a colocarse en la entrada del agua del depósito y cerca de la represa considerando solamente que sea depositada arena, puede llegar a un altura de casi 60 metros en 10 años, con muy altas concentraciones podría dañar los equipos de generación de energía (ALAM,2007), con el paso del tiempo esa arena junto con los fenómenos climáticos podrán acelerar ese proceso, en ambos casos, podrían ocurrir problemas con el canal de aducción (COBRAPE, 2006).

Muchos investigadores bolivianos describen la construcción de la represa Cachuela Esperanza como una forma de preservar los sedimentos que pasan por el río Beni, siendo éste último uno de los más lodosos en el Complejo del Río Madeira, con esto haciendo que la vida útil de las hidroeléctricas de Jirau y Santo Antônio sea prolongada. 


\section{Necesidad energética brasileña}

A través de los años, los países experimentaron grandes cambios importantes en el sector industrial, haciendo que hubiera un aumento significativo en el consumo de energía. La búsqueda de alternativas para satisfacer esta demanda creciente obligó a los países que anhelaban el desarrollo económico, a llevar a cabo una planificación integrada de la oferta y la demanda de energía para que no se agotaran los recursos energéticos. En Brasil, por ejemplo, el gobierno prevé una demanda de energía para el 2012 alrededor de $124.000 \mathrm{MW}$ y de $83.000 \mathrm{MW}$ en el 2002, que corresponde a un crecimiento medio anual del $4,1 \%$.

Debido a ese rápido crecimiento industrial y económico que Brasil ha enfrentado en las últimas décadas, hubo un aumento en el consumo de combustibles fósiles (de origen no renovable), especialmente después del descubrimiento y explotación del pre-sal, donde se enfrentan problemas de clase ambiental como la emisión de contaminantes y gases de efecto invernadero, considerados como causantes del calentamiento global.

Por otro lado, Brasil tiene gran disponibilidad de recursos energéticos renovables, lo que permite la realización de un planeamiento enfocado para la diversificación de la matriz energética (CIMA, 2006).

Brasil presenta una matriz predominantemente renovable para la generación de energía eléctrica, donde la energía hidráulica corresponde al $74 \%$ de la oferta y todavía se incrementan las importaciones, podemos decir que el $89 \%$ de la electricidad en Brasil proviene de fuentes renovables, como se muestra en la siguiente gráfica (BEN, 2012).

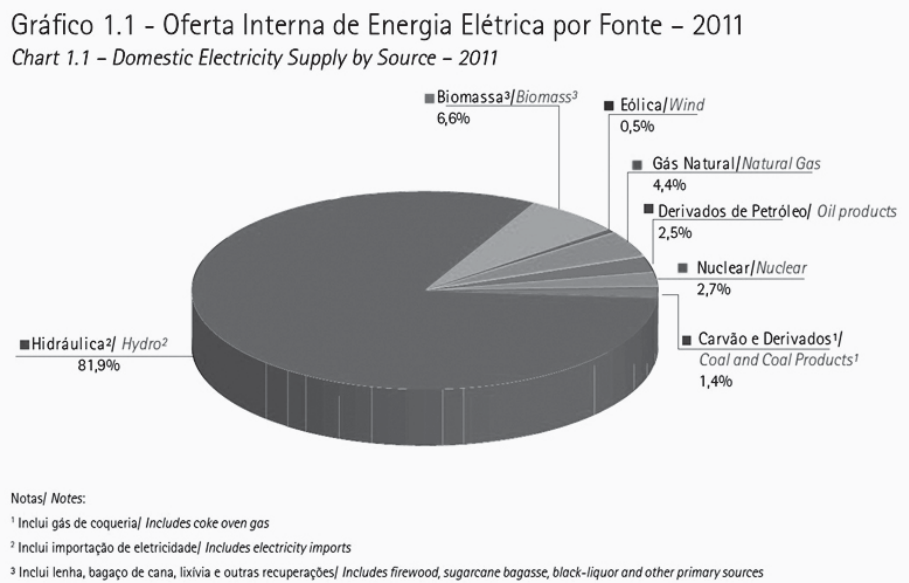

Gráfica 1. La oferta interna de Electricidad.

Fuente: BEN, 2012. 
Sin embargo, podemos afirmar que Brasil tiene una cierta dependencia en la generación de energía hidráulica, y esa dependencia termina generando riesgo en la capacidad ininterrumpida en la distribución de energía debido a su naturaleza incierta de almacenamiento de energía (depósitos). Lo que nos obliga a buscar fuentes alternativas para sustituir la demanda, reduciendo así estos riesgos, como hemos visto, es una fuerte tendencia de generación de electricidad eólica y de pequeñas centrales hidroeléctricas (COBRAPE, 2006).

\section{Interés brasileño en la construcción de Cachuela Esperanza}

La integración energética, que también puede ser vista como un intento de integración económica en los países de América del Sur y como una necesidad de estos países para tener mejores condiciones en el proceso de globalización tuvo inició en 1970 y en 1980 concentrándose en el sector eléctrico. Fue durante este periodo cuando surgió Itaipú (1984) y Salto Grande (1979) entre otros proyectos que involucraron la participación conjunta de diferentes países. También se desenvolvieron políticas energéticas para el suministro y transporte de energía, contribuyendo al proceso de integración, desarrollo sustentable (SUÁREZ, GUERRA, UDAETA, 2006).

En los años 1990 a 2000 se firmaron varios acuerdos internacionales entre los países vecinos de América del Sur, de los cuales Brasil busco beneficiarse de energía eléctrica a bajo costo tomando la función de ampliar la integración eléctrica entre los diferentes países (MOURA, LEGEY, PEREIRA, 2012).

En la perspectiva presentada de conseguir energía a bajo costo, Brasil que tiene una amplia experiencia en la construcción de hidroeléctricas, debido a su potencial geográfico de sus contenedores hidrográficos para la producción de electricidad y con el programa de incentivos para la aceleración del crecimiento (PAC) - donde la autonomía energética es uno de sus pilares para conseguir mantener el crecimiento deseado en el país demuestra intereses en la construcción hidroeléctrica y del complejo de Madeira, con la construcción de las hidroeléctricas de Santo Antônio, Jirau y Cachuela Esperanza que incluyen:

- Atender la demanda de energía, convirtiéndose en independiente y eliminando gradualmente la importación de gas e hidrocarburos;

- La apertura de los mercados del oeste de América del Sur y países asiáticos, especialmente China y la India;

- Aumentar la presencia brasileña a nivel geopolítico, facilitando el acceso a la energía de sus empresas y centros industriales;

- Convertir a los ríos navegables facilitando el transporte de mercancías (MOURA, LEGEY, PEREIRA, 2012). 
Debido a las necesidades energéticas mundiales asociadas al desenvolvimiento Brasil se encuentra en la necesidad de obtener energía a bajo costo. Una alternativa a este problema es la integración con otros países, a través de la construcción de hidroeléctricas, viendo que América del Sur es considerada una región de importancia en el escenario mundial por oferta y disponibilidad de los recursos hídricos. Sin embargo, una serie de problemas y conflictos surgen de estas integraciones en las cuales Brasil es visto por muchos como oportunista, siendo ejemplo significativo los incentivos para la construcción de Cachuela Esperanza.

La construcción de Cachuela Esperanza, traerá serios problemas a Bolivia que no afectarán a Brasil como la necesidad de reubicación de la población local, siendo que parte de esta población está constituida por indios y pérdida de biodiversidad. Además de eso, Bolivia deberá vender a bajo costo la energía generada para poder exportar los excedentes. Desde esta perspectiva, por lo tanto, Brasil va contra las políticas de necesidad de desarrollo sustentable y de planificación integrada. 


\section{Bibliografía}

Alam, Sudam (2007), Projeto Rio Madeira Estudos Hidráulicos e de Sedimentos Relatório Preliminar. Ministério de Minas e Energia Brasília - DF - Brasil, Janeiro.

Arias, G.L.B. (2011), Represa CachuelaEsperanza: posiblesconsecuencias sócio econômicas y ambientales em suconstrucción. Centro de Investigación y Promoción del Campesinado, La Paz.

Bermann, C. (2007), Impasses and controversies of hydroelectricity. Estudos avançados, 21 (59), 139-153 [cited 2012-11-19]. Disponible: http:// www.scielo.br/scielo.php? script=sci_arttext\&pid= S01030142007000100011\&lng=en\&"\&tlng=pt.\%20http://dx.doi.org/ 10.1590/S0103-40142007000100011, acceso en 19, Nov. 2012.

BIC-Bank InformationsCenter (2009), Usinas do Madeira: impactos e ações em terras bolivianas. Disponible:http://www.bicusa.org/es/ Article.11411.aspx.

BRASIL, L. (1986), Resolução CONAMA nº 001, de 23 janeiro de 1986, Dispõe sobre as diretrizes gerais para uso e implementação de Avaliação de Impacto Ambiental. Brasileira. Diário Oficial.

Carpio, M. J. (2008), “Hidrologia e Sedimentos”. In Águas Turvas: alertas sobre as conseqüências de barrar o maior afluente do Amazonas/Gle, São Paulo.

CEADESC. (s.f.), Em Atlas de Megaproyectos de Infraestructuraen Bolivia. Disponible: http://www.ceadesc.org/wp-content/uploads/2011/12/ Atlas_IIRSA11.pdf

Cima, F. M. (2006), Utilização de Indicadores Energéticos no Planejamento Energético Integrado. (Dissertación de Master,Universidad Federal de Rio de Janeiro).

COBRAPE - Cia. Brasileira de Projetos e Empreendimentos (2006), Relatório de Análise do conteúdo dos estudos de Impacto Ambiental (EIA) e do relatório de Impacto Ambiental (Rima) dos Aproveitamentos Hidrelétricos de Santo Antônio e Jirau, no rio Madeira. Ministério Público do Estado de Rondônia. Estado de Rondônia, Dezembro.

Collazos, M. V., Aztarain V. M., Aubareda, P. R. (2010), Un caso de anti cooperación española “El complejo del río Madera”. ObservatoridelDeute em la Globalització

De Sousa, W. L. (2000), Impacto Ambiental de Hidrelétricas: Uma Análise comparativa de duas abordagens (Dissertación de Master,Universidad Federal de Rio de Janeiro). 
EMPRESA, D. P. E. (2012), Balanço Energético Nacional 2012. Disponible: https://ben.epe.gov.br/, visitada en 20 de Nov. 2012. P. Audebert, P. Hapiot, J. Electroanal. Chem. 361 (1993) 177.

ENDE, Empresa Nacional de Electridad (2007), Proyecto hidroelétrico Cachuela Esperança.

Fearnside, P. M. (2006), “Parecer Técnico sobre Ecossistemas”. In COBRAPE. Pareceres dos Consultores sobre o Estudo de Impacto Ambiental do Projeto para Aproveitamento Hidrelétrico de Santo Antônio e Jirau, Rio Madeira - RO. Porto Velho: Cobrape - Cia. Brasileira de Projetos e Empreendimentos / Ministério Público do Estado de Rondônia vol. I.

FOBOMADE (2008), Foro Boliviano sobre Medio Ambiente y desarrollo. Complejo Del Madera.

Goldemberg, J. \&Lucon, O. (2008), Energia, Meio Ambiente e Desenvolvimento ( $3^{\mathrm{a}} \mathrm{ed}$.) (pp.66-69). - rev. ampl. - Editora de la Universidad de São Paulo, São Paulo.

Guyot, J. L., Bourges, J., Hoorelbecke, R., Roche, M. A., Calle, H., Cortes, J., \&Guzman, B. (1988), Exportation de matièresensuspensiondes Andes versl'Amazonie par le Rio Béni, Bolivie.

Guyot, J. L.; Jouanneau, J. M. \&Wasson, J. G. (1999), “Characterisation of river bed and suspended sediments in the Rio Madeira drainage basin” (Bolivian Amazonia). Journal of South American Earth Sciences.12(4), 401-410.

Hinrichs, R.A.; Kleinbach, M. \& Reis, L.B. (2010), Capitulo 1. Hinrichs, R.A.; Kleinbach, M. \& Reis, L.B (Eds) Energia e meio ambiente (pp.3-7). Cesgage Learning, Câmara Brasileira de Livro, São Paulo.

IIRSA. Iniciativa para a Integração da Infra-estrutura Regional Sul-americana.

Laats H. (2009), El Dilema Amazónico “La Construcción de Mega-represas en el Río Madeira”. CEADESC, Bolivía.

Lima, B. W. F. (2009), Centrais Hidrelétricas de Pequeno Porte e o Programa Brasileiro de PCHs.

Moraes, R. A. (2011), “Impactos na Bolívia do desenvolvimentismo brasileiro: o caso de Cachoeira Esperança”. In $36^{\circ}$ Encontro Anual da ANPOCS.

Moura, G. N. P., Legey, L. F. L., Pereira Junior A. (2012), O Diagnóstico do Sistema Elétrico de transmissão brasileiro relacionado á comercialização de Energia Elétrica com países da América do Sul. In XIV Congresso Brasileiro de Energia. Rio de Janeiro. 
PCE, (2005), Engenharia. Estudos Sedimentológicos do rio Madeira.

Pinheiro, M. F. B. (2007), Problemas sociais e institucionais na implantação de Hidrelétricas: seleção de casos recentes no Brasil e casos relevantes em outros países. (Dissertación de master, Universidad Estadual de Campinas)

Pires, S. H. M. (2001), “Planejamento ambiental da expansão da oferta de energia elétrica: subsídios para a discussão de um modelo de desenvolvimento sustentável para a Amazônia”. Parcerias estratégicas, 6 (12).

Porto-Gonçalves, C. W. (2008), “A luta pela apropriação e reapropriação social da água na América Latina”. Oceania, 7 (4.570), 2-510.

Ribera Arismendi, M. O. \& Liga de Defensa del Medio Ambiente (2010), "Mega represas y energía represas del Madeira, Cachuela Esperanza, El Bala y geotermia Laguna colorada actualización (2009-2010)”. Serie de estudios de caso sobre problemáticas socio ambientales en Bolivia.

Soto, G. (2011), Territorio indígena y parque nacional Isidoro Sécure: El final de la ilusión y el advenimiento de lo Nuevo. CEADESC.

Suárez, L. P. L., Guerra, S., \&Udate, M. (2006), “Os Fundamentos Institucionais na integração energética da América do Sul”. InV Congresso Brasileiro de Planejamento Energético (31).

TECSULT. AECOMb (2009), Proyecto hidroeléctrico Cachuela Esperanza.

Tucci, C. E. M.(2007), Análises dos Estudos Ambientais dos Empreendimentos do rio Madeira. Ministério do Meio Ambiente. Instituto Brasileiro de Meio Ambiente - IBAMA.

Recibido: 29.07.2013

Aceptado: 24.11.2014 